\title{
Index Blending: Enabling the Development of Definitive, Discipline-Specific Resources
}

Index Blending is the process of database development whereby various components are merged and refined to create a single encompassing source of information. Once a research need is determined for a given area of study, existing resources are examined for value and possible contribution to the end product. Index Blending focuses on the quality of bibliographic records as the primary factor with the addition of full text to enhance the end user's research experience as an added convenience. Key examples of the process of Index Blending involve the fields of communication and mass media, hospitality and tourism, as well as computers and applied sciences. When academia, vendors, subject experts, lexicographers, and other contributors are brought together through the various factors associated with Index Blending, relevant discipline-specific research may be greatly enhanced.

A s consumers, when we set out to make a purchase, we want the utmost in quality, and when applicable, quantity, and of course all of the other "appeal" factors that might be associated with a given product or service. These factors may include any number of categories, not the least of which is price. In other words, let it suffice to say that, as buyers, we want to have our cake and eat it, too. But how often is this a realistic approach to evaluating a given item for purchase? We first must decide what is important to us, decipher the order of this importance as we see it, and evaluate our options. Wouldn't it be much easier if one product in every situation had all of the factors that we deem important, and the appropriate price to go along with it?

According to Veliyath and Fitzgerald in an article published in Competitiveness Review, firms can either position themselves at the high end, offering higher quality at higher prices, or at the lower end, offering lower quality at a lower price (or anywhere in-between on the continuum of constant value for customers). Customers, however, want more of what they value, such as convenience, speed, state-of-the-art design, quality, etc. Competitors then try to differentiate themselves from their rivals along the same line of constant value, either by offering a higher quality at the same price or the same quality at a lower price (thereby increasing value for the customer). ${ }^{1}$

As such, and using a common example, is it possible to have the handling of a BMW sports car, the luxurious ride of a Cadillac, the passenger space of a Winnebago, the cargo space of an oversized pick-up truck, all for the price of an economy car? It's doubtful. But through recent developments in the electronic research database market- place, and a process known as "Index Blending," we may be closer than ever to this ideal formula when it comes to Web-based reference resources for academic libraries.

The phrase "Index Blending" is used here to describe an original concept/methodology initiated by EBSCO Publishing (EBSCO). This is not to say that EBSCO is the first vendor ever to have combined resources to create a new product, but to the authors' best knowledge, no other vendor has pursued the "blending" of resources to the same extent and with such a strong guiding directive as EBSCO has.

Index Blending is the combining of niche indexes and other important components to create a single definitive index for a particular discipline. As vendors seek to offer the most powerful research database for a given area of study, the pieces may come together through a combination of existing resources and proprietary development. In other words, in order to refine the tools used for research in a discipline, existing resources may be combined, fleshed out, further expanded upon, and enhanced to culminate in the archetypical index for the particular discipline. Perhaps this represents the solution to the dilemma that "database choices become increasingly complex when multiple sources exist that cover the same discipline." ${ }^{2}$

The idea may seem elementary, but the process, however, can be arduous. Processes involved with Index Blending expand upon the basic development stages associated with creating a research database from "scratch," coupled with an increase in applicable factors, which become evident when several existing and emerging resources are involved and subsequently interwoven. As is always the case, the first step to building a solution is to identify the problem and/or the need. In database development, this is, in a nutshell, pinpointing a subject area of research that is lacking a corresponding definitive index, and where study patterns and research interest dictate a need for such a resource. This involves not only conducting surveys and engaging in discussion with advisory boards, librarians, subject experts, users, etc., but also taking a close look at the research resources that are currently available to determine value. Because the process begins with the fact that there is a problem (no definitive index for the particular area in question), the idea is to understand the strengths of available resources, as well as to identify weaknesses.

Through this research process, vendors can further identify independent elements of each resource that may

Sam Brooks (sbrooks@ebscohost.com) is the Senior Vice President of Sales \& Marketing for EBSCO Information Services. Mark Herrick (mherrick@ebscohost.com) is the Vice President of Business Development for EBSCO Publishing. 
provide significant benefit or value, as well as pinpoint the additional important pieces that are not represented in any of the available resources. In both cases (available and not available), these elements may represent various aspects associated with a research index such as content coverage (both current and backfile), quality of indexing and abstracts, software/search functionality, thesauri, etc. Once the identification and research has taken place, vendors should have the necessary knowledge to proceed to the production phase.

Figure 1 helps to illustrate how the Index Blending process can help to develop a new database that fuses together the strengths of existing resources while simultaneously compensating for any individual weaknesses that they may have.

If value is attributed to currently available databases, then, if appropriate, database acquisition may come into play. This is often a critical phase of the process, and may involve the acquisition of more than a single index. However, the desire by a vendor to acquire a given resource is based on several motivating factors, including the quality of the database as a whole, the depth and breadth of its coverage, and at times, the extreme quality of an intricate aspect of a database, which will eventually be said database's contribution to the process of index blending, thus representing its "mark" on the final product.

Because there is no authoritative resource available for a given subject area does not mean necessarily that certain aspects of existing resources are not of utmost quality. Hence, utilizing strengths of existing resources makes sense so as to not "reinvent the wheel" when applicable. In a Journal of Academic Librarianship article discussing the research environment in libraries and the simultaneous utilization of existing library resources,

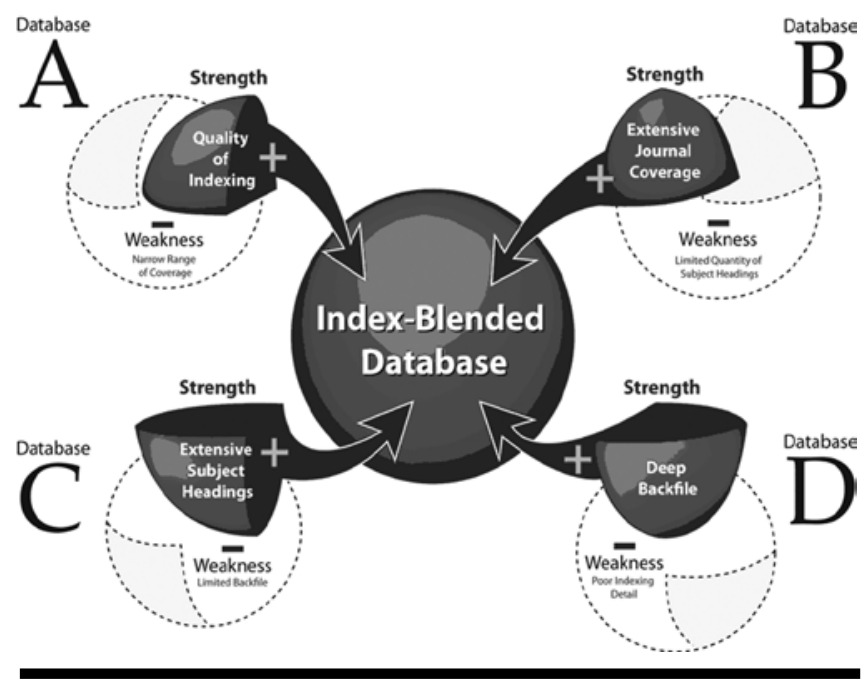

Figure 1. The index blending process similar principles to those used in Index Blending are apparent. "Properly combining library resources to function collectively as a cohesive, efficient unit is the basis of information integration. ${ }^{\prime 3}$ Similar themes to those associated with information integration run through Index Blending. This is attributed largely to the fact that the basic goal of each is to enable the extraction and utilization of essential material pertinent to specific research so as to enhance the overall research process.

\section{The process of Index Blending}

\section{An example}

An interesting example of Index Blending utilized for a major area of study is in the case of communication and mass media. An article in Searcher outlined the development process and release of the database, Communication $\mathcal{E}$ Mass Media Complete, which may be the quintessential instance of the power brought about through Index Blending. In the article, the author first identifies the problem/need as such:

When a communication studies student approaches my reference desk, it can take a few moments before I choose a database to search. Why the delay? Well, to be perfectly blunt, the communication studies literature is all over the place. If the question relates to an aspect of the communications industry, I will often begin with a business database. If the question concerns the effects of media violence on children, I may choose to search one or more of the following: ComAbstracts, PsychInfo [sic], Sociological Abstracts, ERIC, and even a few large aggregators, such as WilsonWeb's OmniFile and EBSCO's Academic Search Premier. In addition, there is the question of finding a single database that covers the communication science and disorders field and the more mass media-focused communication studies field. The result has been a searching strategy that relies on consulting multiple databases-a strategy that may not please impatient or inexperienced patrons.

The need for such an assortment of databases is symptomatic of the discipline. The field of communication studies is extremely interdisciplinary. The discipline's roots began in the study of rhetoric and journalism and now encompass subjects ranging from political communication to film studies to advertising to journalism to communication disorders to digital convergence and to every manner of media. The discipline has strong roots in the social sciences, but also draws heavily on the humanities and the sciences. As some have put it, there is an aspect of communication studies in every discipline. This leaves librarians with the difficult task of finding a single database that covers this wide-ranging discipline. Enter EBSCO's new Communication $\&$ Mass Media Complete database. ${ }^{4}$ 
This overview of the need for a comprehensive resource in areas related to communication and mass media is indicative of the type of information that vendors must extract when deciding their course of action for creating (or not creating) a database to meet such needs. In this instance, the need became apparent to EBSCO upon conducting investigative research in this direction. There were certainly important, quality resources available covering some of the subject areas and subdisciplines, but not a single, all-encompassing resource. Hence, the table was set to move forward and begin the process of database development using the process of Index Blending.

Once the need for a comprehensive communication and mass media database was established, EBSCO began the phases of looking closely at available resources and gathering specific important details about what was required to develop such a database. In order to understand the finer details and make appropriate forward progress in formulating an index for a given research area, a dedicated group of subject experts (advisory board, indexers, lexicographers, etc.) must be established. In addition, aggregators must develop appropriate relationships and key partnerships. In the case of the database Communication $\mathcal{E}$ Mass Media Complete, EBSCO worked diligently to assemble a panel of experts to provide direction. Often, suggestions made by advisory board members ultimately led to larger organizational partnerships.

The first of EBSCO's major partnerships for the benefit of the development of Communication $\mathcal{E}$ Mass Media Complete was with the National Communication Association (NCA). NCA is the oldest and largest national organization to promote communication scholarship and education. Founded in 1914, the NCA is a nonprofit organization of approximately 7,100 educators, practitioners, and students who work and reside in every U.S. state and more than twenty countries. The purpose of the association is to promote study, criticism, research, teaching, and application of the artistic, humanistic, and scientific principles of communication. NCA is a scholarly society and, as such, works to enhance the research, teaching, and service produced by its members on topics of both intellectual and social significance. Staff at the NCA national office follows trends in national research, teaching, and service priorities. It relays those opportunities to its members and represents the academic discipline of communication in those national efforts. ${ }^{5}$

In addition to providing insight and advice into the areas associated with communication and mass media, NCA found in EBSCO an ideal partner to further the tremendous efforts the organization had put into its database, CommSearch. CommSearch, in its original form, was a scholarly communication database with deep, archival coverage of the journals of the NCA and other major journals in the field of communication studies. The database provided bibliographic and keyword references to twenty-six journals in communication studies with coverage extending to the inaugural issue of each-some from as far back as the early decades of the twentieth century. The database also included cover-to-cover indexing of the NCA's first six journals (from their first editions to the present) and author-supplied abstracts from their earliest appearance in NCA journals. As EBSCO's goals were in line with the NCA in terms of improving scholarly research in areas surrounding communication as well as enhancing the dissemination of applicable materials, a partnership was formed, and EBSCO acquired CommSearch. The company acquired this database with the intent to enhance the collection through content additions such that it would take residence immediately as a core component of Communication $\mathcal{E}$ Mass Media Complete.

The second major database acquisition came about similarly to the CommSearch arrangement; only this time, EBSCO worked closely with Penn State University, the developers of a database called Mass Media Articles Index. Created by Jack Pontius and maintained by the Penn State Libraries since 1984, Mass Media Articles Index provided citation coverage for over forty thousand articles on mass media published in over sixty research journals, as well as major journalism reviews, recent encyclopedias, and handbooks in the area of communications studies. This database, which was once a stand-alone research tool, is a good example of how a good-quality resource can arise out of the passion and unique vision of an individual, yet never fully develop into its full potential due to a lack of funding, dedicated staff, and experience in database publishing. Seeing the incredible potential of Mass Media Articles Index, EBSCO earmarked this database as the second major component in its larger communication and mass media product.

As mentioned, the basic idea with Index Blending is to pinpoint the best and most important aspects of each database to carry forward into the final product. It is at this point that difficulty typically arises in the normalization of data. Once core database components are determined, a vendor's expertise in building databases, standardizing entries, etc., comes to the forefront. Furthermore, because another basic ingredient to the process of Index Blending revolves around additional material included by the database developer, that aggregator has the burden of taking the core building blocks of the database and elevating these raw materials to the point where their combination and refinement become the desired end result - a definitive, cohesive index to research in the subject area.

With this in mind, EBSCO carefully selected the indexing components of each resource that were essential to carry forward and substantially expanded the 
abstracting and indexing coverage of appropriate journals in CommSearch and Mass Media Articles Index. The company also added indexing and abstracts for many more of the important titles in the communication and mass media fields that were not covered by these databases. Through its initial research, EBSCO gained a thorough knowledge of which journals and other content sources were not covered by the two acquired databases, and worked to provide coverage for those missing sources. As such, the idea with this database was to cover all appropriate, quality titles indexed in all other currently available communication and mass media-specific databases combined, as well as other important journals not previously covered by any such database. Further still, the company took the database to new levels through the creation and deployment of features such as searchable cited references and index browsing.

Figure 2 provides a visual interpretation of the elements associated with this particular example of Index Blending.

Often academic librarians consider aggregated full-text databases as a means for accessing full-text information quickly, but with a negative outlook toward the quality of the indexing included in these databases. However, it is EBSCO's intention to create first and foremost a powerful index, such that any full text included is that much easier to locate and utilize. According to Cleveland and Cleveland in the book Introduction to Indexing and Abstracting, 3rd ed., "In any retrieval system, success or failure depends on the adequacy of the indexing and the related searching procedures." ${ }^{\prime \prime}$ EBSCO wholeheartedly agrees with this statement. And though the company is the leader in providing full-text databases, it continues to raise the bar for these databases through not only constantly increasing the quality and quantity of full text, but also by enhancing indexing, abstracts, and associated search functionality. A database may provide the greatest collection of full text, yet it is still only as good as its underlying indexing framework that guides users to the appropriate content. Index Blending allows for this ideal because the development of the indexing takes place at the onset as the primary objective, and full text may be included at a later stage. This is precisely the case with EBSCO's communication/communications database where the first iteration of the collection (Communication $\mathcal{E}$ Mass Media Index) did not include full text, and the Complete (full-text version) was soon to follow.

Thus, in the case of Communication \& Mass Media Complete, once the core elements for the index were in place, refined, and normalized, EBSCO moved forward

in the area of full-text content. In addition to the inclusion of full text for all of the NCA journals, which David Oldenkamp refers to as "heavyweights in communication studies," EBSCO included full-text coverage for nearly 230 titles. According to Oldenkamp, as of April 2004, the competing database with the next largest number of publications covered in full text included only sixteen full-text titles.

Though Index Blending is not the traditional way in which to build a database, and may actually be the most labor-intensive way in which to proceed, the end results can be remarkable when done properly. Using this process, "EBSCO has managed to create the largest and most comprehensive database serving the needs of communication studies scholars, faculty, students, and librarians." ${ }^{8}$ In addition, a review published in The Charleston Advisor determined that "EBSCO has brought together two reliable but atrophied resources and refreshed them with new search capabilities and added content, such as abstracts. These have been combined with a healthy dose of 'not indexed anywhere' new titles and interdisciplinary sources to create a comprehensive 
resource that will satisfy the needs of students, faculty, and researchers. ${ }^{\prime \prime}$

\section{Another example of Index Blending}

\section{Hospitality \& Tourism Index}

Index Blending is a concept as much as it is a process and a means to an end. Much like applying a particular theory to a number of different instances, Index Blending is inter-disciplinary in application. Thus, the area of communication/communications as described previously, is simply an example of practical implementation of this concept, and a particular way in which the process was approached given the specific elements involved. Another discipline to which Index Blending has been applied is the niche areas related to hospitality and tourism.

According to Professor Vivienne Sario, Director of Travel and Tourism at Community College of Southern Nevada, "On a global basis the hospitality and tourism industry employs more than 10 percent of the worldwide workforce. It contributes over $\$ 4$ trillion in gross global output. This means travel and tourism is the world's largest industry." 10 Though still considered (perhaps incorrectly) a "niche" area of study, the number of hospitality and tourism programs supported in colleges and universities around the globe has also increased to the point where dozens and dozens of two- and four-year academic institutions provide related courses of study.

From a business perspective, in order to justify the amount of resources that would inevitably be expended to develop a high-end, comprehensive database, the basic criteria needed for database development must first be in place. Considering the economic vastness of the hospitality and tourism industry, the interest and research need is quite apparent. If there is at least one clearly definitive academic resource covering the subject area, in all likelihood, the decision would be made to cease exploration and development in that area. Contrarily, when EBSCO conducted exhaustive research to determine the need for a new index to literature in the areas of hospitality and tourism, the unanimous conclusion was to move forward in the development of a product that would go above and beyond the level of the existing resources. This is not to say that quality was not inherent in some of the existing resources. In actuality, the fact that there were already quality (albeit perhaps incomplete) resources available, paved the way for utilizing principles of Index Blending in the development of a more comprehensive resource.

The first element of what was to become EBSCO's Hospitality \& Tourism Index was Purdue University's Lodging, Restaurant, $\mathcal{E}$ Tourism Index (LRTI). As an indicator of the level of emphasis attributed to this subject area by the university, Purdue's hospitality and tourism management undergraduate program was ranked number one nationally by a survey published in the Journal of Hospitality $\mathcal{E}$ Tourism Education. ${ }^{11}$ A previous survey conducted by the same journal used a different methodology and sample, but still ranked Purdue's hospitality and tourism management (HTM) program number one in the nation. ${ }^{12}$

To provide insight into the Purdue HTM program, the origins and history of LRTI, the need for a comprehensive database, and the university's decision to work with EBSCO, questions were asked of two prominent Purdue faculty members: Raphael Kavanaugh, Head, Hospitality and Tourism Management Department, and Priscilla Geahigan, Head, Consumer and Family Sciences Library. The following is taken from e-mail correspondence among one of the authors (Sam Brooks), Kavanaugh, and Geahigan:

BROOKS: How long has Purdue offered a Hospitality \& Tourism Management program?

KaVANAUGH: The program began in 1928 as the Department of Institutional Management.

BROOKS: When and why did Purdue decide to create the Lodging Restaurant $\mathcal{E}$ Tourism Index (LRTI)?

KAVANAUGH: To fill a serious void of access to relevant research conducted related to the industry.

GEAHIGAN: Before 1990 coverage of the hospitality industry within business indexes and databases was limited. To meet the needs of researchers and students, Purdue's Restaurant, Hotel, Institutional, and Tourism Management Department, an in-house indexing project, started in the Purdue Consumer and Family Sciences Library in 1977. Citations of articles from scholarly and trade journals were entered on index cards, filed by subject headings. In 1985 the project became more formalized and migrated into partnership with a few other academic institutions. A printed index titled Lodging and Restaurant Index started. In 1987, Purdue became the sole producer of the Index. In 1995, the Index was renamed the Lodging, Restaurant, and Tourism Index (LRTI), with expanded scope and coverage. Over the years, data diskettes and CD-ROM formats were added to the printed version.

BROOKS: How important are "niche" or subject-specific databases to support research in a given area such as H\&T?

GEAHIGAN: In contrast to earlier years, students can now get their information from a multitude of databases and 
venues. At Purdue, we have databases that cover all aspects of business and management. Undergraduate students often get confused and impatient at the large number of databases offered. A subject specific database like HTI gives them a place to start without feeling lost.

BROOKS: Why did Purdue decide to partner with EBSCO, and subsequently merge $L R T I$ in the larger Hospitality $\mathcal{E}$ Tourism Index (HTI)?

GEAHIGAN: We realized that we do not have the resources to support a database that measures up to industry technology standards and have long decided to look for a company to take over LRTI. EBSCO's offer was attractive to Purdue because of their willingness to assume future indexing of the LRTI journals. In addition, many Purdue students are already familiar with the EBSCO interface because we have numerous other EBSCO hosted databases. We are pleased that LRTI became the foundation of EBSCO's building of HTI. ${ }^{13}$

The second foundational component of the database also came about through acquisition from an academic institution. Articles in Hospitality and Tourism was coproduced by Oxford Brookes University and the University of Surrey. Bournemouth University was also a source of data for this database between the years of 1988 and 1998. This database provided details of more than forty-six thousand English-language articles selected from more than 330 relevant academic and trade journals published worldwide from 1984 to $2003 .^{14}$

Rounding out the list of three existing resources that were acquired by EBSCO, the Hospitality Database (acquired from the original developers at Cornell University) was also assimilated into the new hospitality and tourism database. The Hospitality Database evolved from the print publication Bibliography of Hotel Management and Related Subjects that was originally established in the 1950s by Blanche Fickle, the first director of the library at Cornell University's School of Hotel Administration. ${ }^{15}$ This database, founded on the vision of Ms. Fickle, would serve as a core resource for EBSCO's new Hospitality $\mathcal{E}$ Tourism Index by providing it with a foundation of quality indexing for journals related to the study of hotel administration and management.

EBSCO completed the initial development of its hospitality and tourism database by reviewing applicable subscription statistics maintained by its sister company, EBSCO Subscription Services, in order to locate other publications relevant to the various subdisciplines of hospitality and tourism. Any such publications that were not already indexed by the other three existing resources were targeted for inclusion in the new Hospitality $\mathcal{E}$ Tourism Index.
Figure 3 provides a visual interpretation of the elements associated with this particular example of Index Blending.

Following the initial release of Hospitality $\mathcal{E}$ Tourism Index, in order to provide an even more inclusive research experience, EBSCO proceeded to develop and release a full-text version of this resource entitled Hospitality $\mathcal{E}$ Tourism Complete. This new variant of the database offers users the same indexing infrastructure as Hospitality $\mathcal{E}$ Tourism Index, as well as provides the additional benefit of immediate access to relevant full-text content. While the availability of full text is certainly of immense value, it is still the quality of underlying indexing that allows this database to be regarded as truly innovative. In fact, this same perspective was echoed in a recent review in CHOICE where the author states that "Hospitality $\mathcal{E}$ Tourism Complete indexes its specialized subject area better than any other product currently available."16

\section{The whole is greater than the sum of its parts}

The process of Index Blending not only brings together content from a variety of resources, it also has the power to increase the research value of that same content. By combining such content under the umbrella of a single comprehensive database, pertinent information can now be more efficiently accessed and cross-referenced with other relevant content. Previously, the same body of information could only be explored via a highly ineffective, piecemeal research process.

One last example that demonstrates this potential increase in research value is found in the Computers $\mathcal{E}$

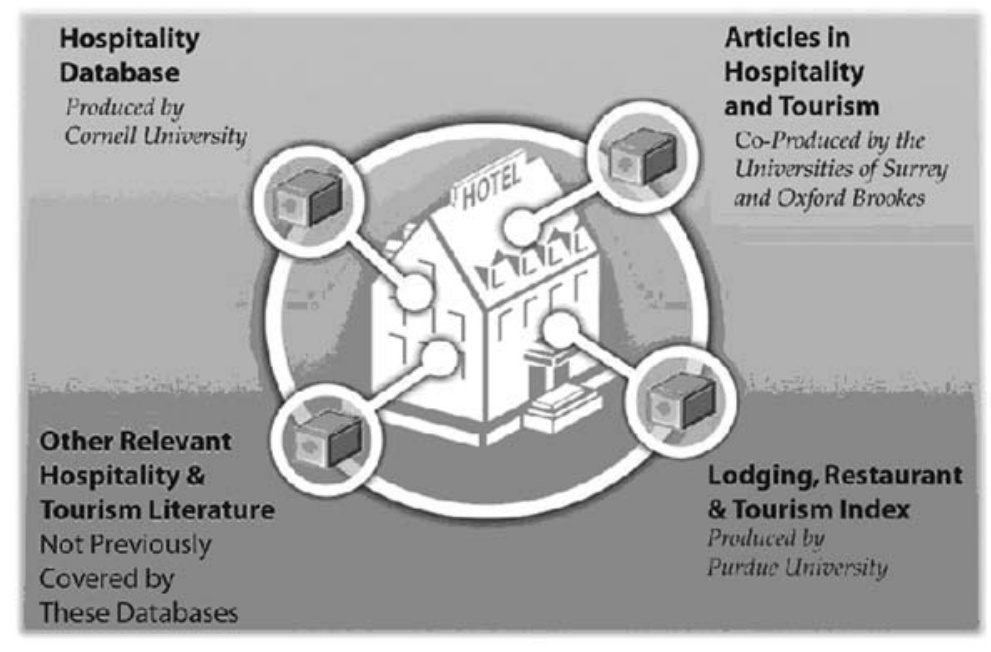

Figure 3. Indexing components of Hospitality \& Tourism Complete 
Applied Sciences Complete database. This resource was shaped through the acquisition and merger of three distinct indexes-Computer Science Index (CSI), Internet \& Personal Computing Abstracts (IPCA), and Information Science $\mathcal{E}$ Technology Abstracts (ISTA) - and rounded out with additional indexed content relevant to the larger discipline. This resulted in a total of 1,100 active journals indexed back as far as 1965 . Then, after two years of dedicated licensing work with publishers, full text for more than 570 of those titles was added to provide more direct access to such content for researchers.

Figure 4 illustrates how the various subject areas (unique and shared) covered by the three original databases were merged together in the blending process.

From this diagram, it is apparent that the original three databases were already quality resources in their own right and adequately represented their respective subject areas. However, it should also be apparent that, through the process of Index Blending, the value of the original databases has been enhanced via the fusion of their unique, yet complementary content into a single comprehensive resource.

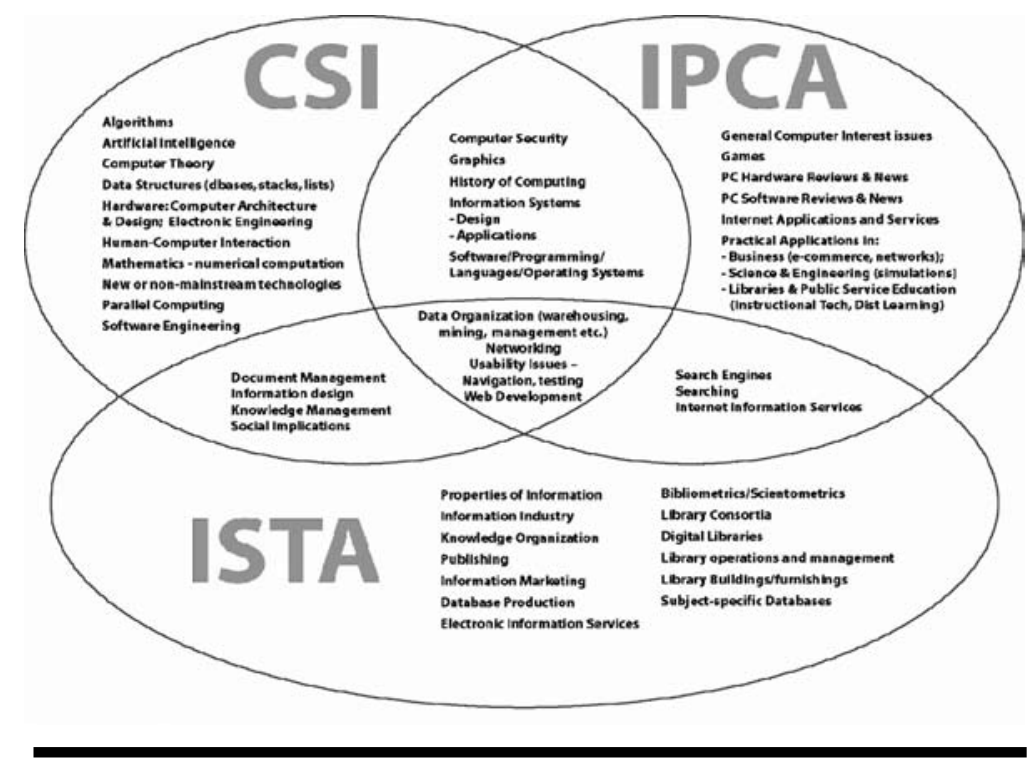

Figure 4. Subject areas of component databases are merged into a cohesive whole through index blending

index for their respective subject area(s). Therefore, when a company implements the practice of Index Blending for some of its products, the resulting effects are twofold. The databases created directly as a result of the Index Blending process are the first to benefit, and the company's other databases (including those with full text) may also benefit from Index Blending in an indirect manner. In the end, however, the success of any Index Blending initiative is measured by the level of benefit that it provides to applicable researchers and other users of the resulting databases.

$\mathcal{E}$ Applied Sciences Complete represent only three of several subject-specific databases culminating from the process of Index Blending, most database producers (including EBSCO) would likely agree that this is not a common procedure for database development. However, the knowledge that a company derives from the process often has a significant impact on the company's other, "nonblended" databases. Index Blending typically requires a high degree of refinement in order to be fully successful, so when a company engages in this rigorous developmental process, the newfound experience and expertise gained from it may spill over into the company's other database initiatives. End users may notice improved indexing, abstracts, and other valuable components that are now included in other more established full-text resources from the same vendor. Databases that were once viewed simply as "aggregated full-text databases" may be looked upon in a different light after the company adopts the process of Index Blending for other, unrelated database projects. Though these databases may still provide easy access to an abundance of full-text content, they may also now be considered the definitive

\section{References}

1. Rajaram Veliyath and Elizabeth Fitzgerald, "Firm Capabilities, Business Strategies, Customer Preferences, and Hypercompetitive Arenas: The Sustainability of Competitive Advantages with Implications for Firm Competitiveness," Competitiveness Review 10 (2000): 56-82.

2. M. Suzanne Brown, Jana S. Edwards, and Jeneen LaSeeWillemssen, "A New Comparison of the Current Index to Journals in Education and the Education Index: A Deep Analysis of Indexing," The Journal of Academic Librarianship 25 (May 1999): 216-22.

3. Sam Brooks, "Integration of Information Resources and Collection Development Strategy," The Journal of Academic Librarianship 27 (July 2001): 316-19.

4. David Oldenkamp, "EBSCO's New Communication and Mass Media Complete (CMMC) Database," Searcher 12, no. 4 (Apr. 2004): 40.

5. National Communication Association Web site. http:// www.natcom.org (accessed Aug. 2004). 
6. Donald B. Cleveland and Ana D. Cleveland, Introduction to Indexing and Abstracting, 3rd ed. (Greenwood Village, Colo.: Libraries Unlimited, 2001): 26.

7. Oldenkamp, "EBSCO's New Communication and Mass Media Complete (CMMC) Database."

8. Ibid.

9. Dodie Owens, "Advisor Reviews-Standard Review: Communication and Mass Media Complete," The Charleston Advisor 6, no. 4 (Apr. 2005): 45.

10. Vivienne Sario, "Hospitality \& Tourism Programs," http:/ / www.studyusa.com/articles/hospitality.asp (accessed June 1, 2006).

11. Purdue University Web site. http://news.uns.purdue. edu/UNS / html4ever/030130.Kavanaugh.rank2003.html (accessed June 1, 2006).
12. Michael G. Brizek and Mahmood A. Khan, "Ranking of U.S. Hospitality Undergraduate Programs: 2000-01," Journal of Hospitality \& Tourism Education 14, no. 2 (2002): 4.

13. Raphael Kavanaugh and Priscilla Geahigan, e-mail message with author Sam Brooks, Feb. 3, 2005.

14. Articles in Hospitality and Tourism Web site (hosted by the University of Surrey). http://libweb.surrey.ac.uk/aht2/about .asp (accessed June 1, 2006).

15. Cornell University's School of Hotel Administration Web site. http://www.nestlelib.cornell.edu/history.html (accessed June 1, 2006)

16. S. C. Awe, "Reference-Social and Behavioral SciencesHospitality \& Tourism Complete," CHOICE 43, no. 10 (June 2006). 